\title{
BMJ Open Impact and variability of social determinants of health on the transmission and outcomes of COVID-19 across the world: a systematic review protocol
}

\author{
Minhazul Abedin (D) , ${ }^{1}$ Abrar Wahab, ${ }^{1}$ Farah Naz Rahman (D) , \\ Fardina Rahman Omi, ${ }^{2}$ Saadia Shareen, ${ }^{3}$ Shagoofa Rakhshanda (iD , ${ }^{1}$ \\ Labida Islam, ${ }^{1}$ Cinderella Akbar Mayaboti, ${ }^{1}$ Uttam Kumar Saha, ${ }^{1}$ Fazlay Faruque, ${ }^{4}$ \\ Lauren M Fletcher (D) , ${ }^{5}$ Saidur Mashreky (D) ${ }^{1,2}$
}

To cite: Abedin M, Wahab A Rahman FN, et al. Impact and variability of social determinants of health on the transmission and outcomes of COVID-19 across the world: a systematic review protocol. BMJ Open 2021;11:e053481. doi:10.1136/ bmjopen-2021-053481

- Prepublication history and additional supplemental material for this paper are available online. To view these files, please visit the journal online (http://dx.doi.org/10.1136/ bmjopen-2021-053481).

Received 13 May 2021 Accepted 13 November 2021

Check for updates

(C) Author(s) (or their employer(s)) 2021. Re-use permitted under CC BY-NC. No commercial re-use. See rights and permissions. Published by BMJ.

For numbered affiliations see end of article.

Correspondence to

Minhazul Abedin;

abedin@ciprb.org

\section{ABSTRACT}

Introduction The COVID-19 pandemic has exacerbated health inequalities across the globe, disproportionately affecting those with poor social determinants of health (SDOHs). It is imperative to understand how SDOH influences the transmission and outcomes (positive case, hospitalisation and mortality) of COVID-19. This systematic review will investigate the impact of a wide range of SDOHs across the globe on the transmission and outcomes of COVID-19.

Methods and analysis This review will follow the Preferred Reporting Items for Systematic Reviews and Meta-Analyses Protocol guidelines. We will search three electronic bibliographical databases (MEDLINE via PubMed, Embase and Scopus), as well as the WHO COVID-19 Global Research on Coronavirus Disease database. We will consider observational studies that report statistical relationships between the SDOHs (as listed in PROGRESS-Plus and Healthy People 2020) and COVID-19 transmission and outcomes. There will be no limitation on the geographical location of publications. The quality of included observational studies will be assessed using a modified version of the Newcastle-Ottawa Scale. A narrative synthesis without meta-analysis reporting standards will be used to report the review findings. Ethics and dissemination This review will be based on published studies obtained from publicly available sources, and therefore, ethical approval is not required. We will publish the results of this review in a peer-reviewed journal, as well as present the study findings at a national conference.

PROSPERO registration number CRD42021228818.

\section{INTRODUCTION}

In December 2019, a pneumonia case associated with SARS-CoV-2 was first identified in Wuhan, Hubei province, China. As of 24 April 2021, this emerging infection has caused 154640649 infections and 3232285 deaths globally. ${ }^{1}$ The current experience of

\section{Strengths and limitations of this study}

- This review will address the impact of a wide range of social determinants of health and the geographical variations that affect the transmission and outcomes of COVID-19.

- A multidisciplinary team is involved in this review, and the search strategy was undertaken with the involvement of a library information scientist.

- A standard scientific reporting format will be used to summarise the review findings to avoid the shortcoming and biases of narrative synthesis.

- The influx of journal articles that were published over the last year in response to the current pandemic, without a scientific track record of previous studies, will bereviewed in this article.

the COVID-19 pandemic has taken the world by surprise, despite the modern advances in health sciences. Outcomes of the disease not only have included an increased death toll but also have put a spotlight on health inequalities. $^{2}$ According to the WHO, social determinants of health $(\mathrm{SDOH})$ are the non-medical factors that influence the health outcome of humans. They broadly define it by the conditions in which people are born, grow, work, live and age, and the wider set of forces and systems shaping the conditions of daily life. ${ }^{3}$ All these conditions have a major impact on the health, well-being and quality of life of an individual. ${ }^{4}$ The transmission of any communicable disease is highly dependent on these SDOH. The vast dynamics of disease transmission may result in symptomatic or asymptomatic disease outcomes, which may lead to hospitalisation and/or death. Generally, people residing in socioeconomically 
low-income areas are at least three times more likely to die of preventable diseases compared with those in highincome areas. ${ }^{5}$ The socioeconomically disadvantaged population also has limited access to healthcare services. Studies show that even those who live above the poverty line are more prone to be affected by chronic health conditions. ${ }^{6}$

This pandemic has made it clear that SDOH differentially affect disease transmission and outcomes globally. ${ }^{2}$ Reports and studies generated from countries with high infection rates suggest that geographical variations were signifying higher transmission rates, hospitalisations and mortality in marginalised, densely populated, low-income and crowded households. ${ }^{7-12}$ Populations living in crowded neighbourhoods are at a higher risk of becoming ill and transmitting the virus at an increased rate, as social distancing may not be feasible. ${ }^{13-15}$

Access to healthcare services is described by the National Academies of Sciences, Engineering, and Medicine as the 'timely use of personal health services to achieve the best possible health outcomes' ${ }^{16}$ Access to healthcare is an influential SDOH that has gained attention during this COVID-19 pandemic. ${ }^{17} 18$ Accessibility to healthcare services may include geographical accessibility (within physical reach), economic accessibility (within financial reach) and cultural accessibility (acceptance and communication). ${ }^{19-21}$ Health insurance is an important aspect of accessibility which is evident from several studies and reports. It has been demonstrated that there is unequal coverage of health insurance and access to services, leading to poorer health outcomes in marginalised groups. ${ }^{22}{ }^{23}$ In a study by Gallup and West Health, it was found that $14 \%$ of adults in the USA do not seek medical attention when experiencing fever and dry cough due to the high cost of healthcare. ${ }^{24}$ Given the fact that fever and dry cough are the most common symptoms of COVID-19, there is a high chance that many COVID-19 cases may go unnoticed or undiagnosed, which may result in unknowingly transmitting the disease. ${ }^{25}$ Primary care visits may not be feasible, or people may hesitate to use healthcare resources without health insurance. This inability puts those without health insurance at risk of not being screened for chronic conditions, such as cardiovascular disease (CVD), hypertension, asthma and diabetes. Therefore, during this health emergency, access to healthcare may play a major role in whether an individual will test for COVID-19 and follow recommendations, such as social distancing and other preventive measures. ${ }^{26}$

In terms of racial disparities, the USA has experienced disproportionate outcomes of infection, hospitalisation and mortality among historically marginalised groups, such as African-American, Hispanic and Asian populations. ${ }^{27} 28$ Studies show that mortality due to COVID-19 is associated with socioeconomic class. ${ }^{29-31}$ Black, Asian and minority ethnic backgrounds represented one-third of the intensive care unit (ICU) admission in UK hospitals, while Somali immigrants living in Norway had a 10 times higher rate of infection compared with the non-immigrants. ${ }^{32} 33$ Those

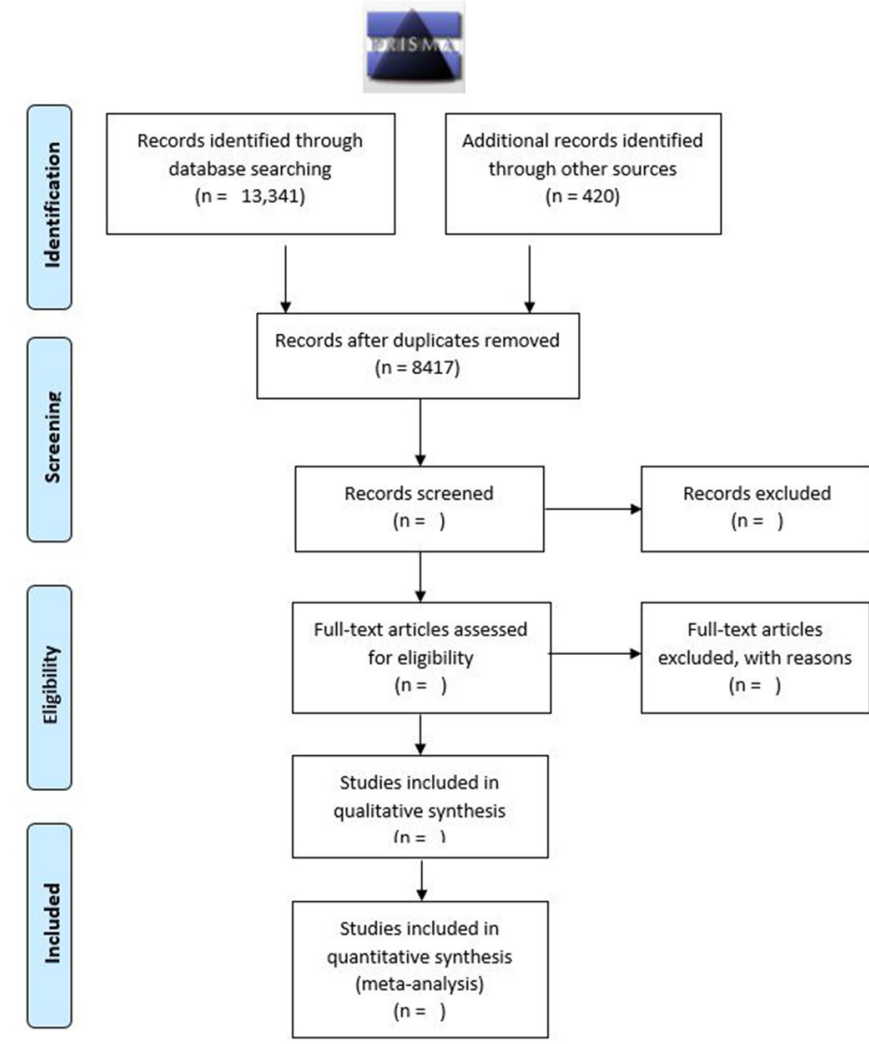

Figure 1 Preferred Reporting Items for Systematic Reviews and Meta-Analyses 2009 flow diagram.

who are minorities may not be aware of their underlying conditions due to barriers in accessing primary care and might be at high risk of developing more severe symptoms during this pandemic. ${ }^{3435}$

It is imperative to focus on SDOH during the COVID-19 pandemic to better understand preventable health inequalities and to improve health outcomes. As such, there is an immediate need to comprehend the social determinants of COVID-19 transmission and outcomes to decrease human suffering and save lives. ${ }^{36}$ In addition to known clinical risk factors, knowledge of social risk factors will help policymakers and healthcare decision-makers to prioritise groups who are most at risk during the COVID-19 pandemic and formulate health messages accordingly.

\section{Objectives}

The objectives of this study are

1. To find the impact of SDOHs on the transmission and outcomes of COVID-19.

2. To explore the geographical variations of $\mathrm{SDOH}$ on transmission and outcomes of COVID-19.

\section{METHODS}

This protocol is registered with the International Prospective Register of Systematic Reviews under the registration number (CRD42021228818) and follows the Preferred Reporting Items for Systematic Reviews and Meta-Analyses guidelines, ${ }^{37}$ as shown in figure 1. 
Table 1 Inclusion and exclusion criteria for the review

\begin{tabular}{ll}
\hline Inclusion criteria & Exclusion criteria \\
\hline $\begin{array}{l}\text { Empirical-based observational quantitative studies such as cross- } \\
\text { sectional, case-control, prospective and retrospective cohort, }\end{array}$ & $\begin{array}{l}\text { Studies in non-English language. } \\
\text { predesign-postdesigns and modelling studies. }\end{array}$ \\
Studies covering any geographical locations. & data), review studies, systematic review, review of the systematic \\
Studies in the English language only. & review, meta-analysis, narrative reviews, conference abstracts, \\
Studies covering people of all age groups. & Pharmacological and biochemical studies, animal model studies, \\
Studies that include SDOH listed in PROGRESS-Plus \& Healthy People & Opinion literature, including commentaries, editorials, brief reports, \\
2020. & perspectives and analyses. \\
Studies that include any COVID-19-positive population. & Studies that include COVID-19 disease outcomes among special \\
Studies that include populations tested positive for COVID-19, with & populations (such as patients with HIV, prisoners, patients with cancer, \\
underlying comorbidities. & etc). \\
Studies that include a population that required hospitalisation or & Articles with missing descriptions of findings and/or results. \\
resulted in mortality due to COVID-19. & \\
\hline
\end{tabular}

$\mathrm{SDOH}$, social determinant of health.

\section{Eligibility criteria}

A summary of eligibility criteria is shown in table 1 .

\section{Types of studies}

The types of studies that will be considered in this systematic review will include empirical quantitative studies following prospective and retrospective cohorts, case-control, cross-sectional, predesign-postdesign and modelling studies that report statistical relationships between exposures of interest (ie, SDOH) and COVID-19 transmission and outcomes. The review will address the potential interaction effect or intersectional approach only if the analysis reports such findings, which is generally found in predictive modelling. Any hypothetical interaction or intersectional findings will be excluded from this review. This review will include studies with both self-reported and directly tested measures. We will only include studies published in the English language. Qualitative studies, reviews, narrative reviews, systematic reviews, meta-analyses, pharmacological and biochemical studies, animal model studies, conference abstracts, conference proceedings and theses/dissertations will not be included in this systematic review. Simulation modelling, which does not report new empirical data, opinion literature, including commentaries, editorials and analyses that discuss COVID-19 regarding equity, social determinants, and/or vulnerable populations, will also be excluded from the review.

\section{Types of study population}

Studies in this review will include populations of all ages, ethnicity, gender and geographical location. We will consider any population that has been tested for COVID-19.

\section{Types of outcome measures}

Studies will be included if they use a measure for SDOH that could be hypothesised to affect the transmission and outcomes (confirmed positive case, hospitalisation and mortality) of COVID-19. We will use Healthy People 2020 and PROGRESS-Plus to classify our definition of SDOH. ${ }^{38}$ Healthy People 2020 addresses five SDOHs: economic stability, education, social and community context, health and healthcare, and neighbourhood and built environment. PROGRESS-Plus refers to the place of residence, race/ethnicity/culture/language, occupation, gender, religion, education, socioeconomic status and social capital. Plus refers to personal characteristics associated with discrimination (eg, age and disability), features of relationships (eg, smoking parents, excluded from school) and time-dependent relationships (eg, leaving the hospital, respite care, other instances where a person may be temporarily at a disadvantage).

\section{Search strategy}

We have developed a comprehensive literature search to locate both published and non-traditional literature. We will search for articles published from 1 January 2019 to 31 December 2021, in three electronic bibliographic databases (PubMed, Embase and Scopus), as well as the WHO Global Research on Coronavirus Disease database, to ensure results focused on the novel COVID-19 pandemic and retrieve preprint articles and non-traditionally published literature. Our COVID-19 search strategy was developed using the published Ovid Embase COVID-19 search strategy translated for use in the searched databases. ${ }^{40}$ The full, detailed search strategy is available in online supplemental file 1. Following the search, all identified citations will be imported into EndNote X9 (Clarivate Analytics, Pennsylvania, USA), and duplicates will be removed. Deduplicated results will be exported to an Excel 2016 spreadsheet for screening. The reference lists of all included studies will then be reviewed for eligible studies.

\section{Study records}

\section{Selection process}

Based on the title and abstract (where available), 10 researchers will initially review and assess potential articles for eligibility that meet inclusion criteria while maintaining a low threshold for consideration for further review. The same researchers will screen the full text of the potential articles for inclusion. We will resolve 
disagreement or uncertainty regarding the eligibility of particular studies through discussion by two independent researchers.

\section{Data management and collection}

Ten researchers will work on data extraction using a piloted data extraction tool, and the extracted data will be reviewed by a supervisor for errors.

Data to be collected include

1. Study details (title, year of publication, author, abstract, study design, country and region).

2. Study population characteristics (age, sex, etc).

3. Sampling strategy and sample size.

4. SODH including participant demographics (place of residence, occupation, race/ethnicity/culture/language, education, religion, social capital, socioeconomic status, economic stability, education, social and community contexts, health and healthcare access, neighbourhood and built environment). ${ }^{38} 39$

5. Outcome severity of COVID-19.

\section{OUTCOMES}

This systematic review will focus on SDOH that impacts the transmission and outcomes of COVID-19. We will also look for the geographical variations of these outcomes from the selected articles for this review. The following outcomes will be examined:

1. Positive COVID-19 cases.

2. Hospitalisation due to COVID-19 that includes regular bed patient admission, high-dependency unit (HDU) patient admission and ICU patient admission.

3. Mortality due to COVID-19.

\section{RISK OF BIAS}

To assess the quality of included observational studies, we will use a modified version of the Newcastle-Ottawa Scale. ${ }^{41}$ Two reviewers will independently assess each study, with discrepancies resolved by agreement or a third independent assessor if such agreement cannot be achieved.

\section{DATA SYNTHESIS AND ANALYSIS}

This review is broad and exploratory in nature, and therefore, a meta-analysis of the effect estimate is not planned, but data will be evaluated for the possibility of conducting a meta-analysis. To avoid the shortcomings in the reporting of narrative synthesis, we will follow the Synthesis Without Meta-analysis reporting standards (SWiM) ${ }^{42}$ We will tabulate the results and narrate the summary of findings for each SDOH identified in this review. Additionally, we will prepare at least three tables consisting of the study characteristics, participant characteristics, a summary of findings that describes study setting, size, methodology, primary outcomes, secondary outcomes, summary statistics and/or statistical results (risk ratios, odds ratios, risk differences, mean differences, standardised mean differences and the ratio of means), and relationship to $\mathrm{SDOH}$.

\section{DISCUSSION}

Our systematic review will focus on the role that $\mathrm{SDOH}$ play in the transmission and outcomes of COVID-19. We hope that the concluded review will provide evidence to determine the impact and variability of SDOH associated with SARS-CoV-2 infection and the dynamics of transmission and outcomes. This protocol will guide the review homogeneously and methodologically. Our systematic review will address a gap in the COVID-19 literature by exploring not only the impact SDOHs have on the transmission and outcomes of the disease but also by exploring the global geographical differences of $\mathrm{SDOH}$ and its impact on the COVID-19 pandemic. Through this review, we hope to provide an empirical understanding of the diverse social determinants of COVID-19, which could appraise the global pandemic response efforts as well as provide comprehensive evidence for future public health policy implications.

\section{PATIENT/PUBLIC INVOLVEMENT}

There will be no patient or public involvement in this study.

\section{ETHICS AND DISSEMINATION}

As the data included in this review have been published or obtained from publicly available sources, ethical approval is not required. We will submit the full systematic review manuscript to a peer-reviewed journal and will present the study results at a national conference.

\section{Author affiliations}

${ }^{1}$ Public Health Sciences, Centre for Injury Prevention and Research Bangladesh, Dhaka, Bangladesh

${ }^{2}$ Noncommunicable Diseases, Bangladesh University of Health Sciences, Dhaka, Bangladesh

${ }^{3}$ Department of Life Sciences, School of Environment and Life Sciences, Independent University, Dhaka, Bangladesh

${ }^{4}$ Department of Preventive Medicine, School of Population Health, The University of Mississippi Medical Center, Jackson, Mississippi, USA

${ }^{5}$ Rowland Medical Library, The University of Mississippi Medical Center, Jackson, Mississippi, USA

Contributors All authors have contributed equally to the design and conceptualisation of this review. MA, AW, FNR, FRO, SS, SR, CAM and LI drafted the manuscript and protocol with UKS, FF, SM and LMF as editors. LMF developed the search strategy with input from UKS, FF and SM concerning keywords and databases. All review methods were planned and discussed with the authors, with LMF acting as a methodological mentor.

Funding The authors have not declared a specific grant for this research from any funding agency in the public, commercial or not-for-profit sectors.

Competing interests None declared.

Patient consent for publication Not applicable.

Provenance and peer review Not commissioned; externally peer reviewed.

Supplemental material This content has been supplied by the author(s). It has not been vetted by BMJ Publishing Group Limited (BMJ) and may not have been peer-reviewed. Any opinions or recommendations discussed are solely those 
of the author(s) and are not endorsed by BMJ. BMJ disclaims all liability and responsibility arising from any reliance placed on the content. Where the content includes any translated material, BMJ does not warrant the accuracy and reliability of the translations (including but not limited to local regulations, clinical guidelines, terminology, drug names and drug dosages), and is not responsible for any error and/or omissions arising from translation and adaptation or otherwise.

Open access This is an open access article distributed in accordance with the Creative Commons Attribution Non Commercial (CC BY-NC 4.0) license, which permits others to distribute, remix, adapt, build upon this work non-commercially, and license their derivative works on different terms, provided the original work is properly cited, appropriate credit is given, any changes made indicated, and the use is non-commercial. See: http://creativecommons.org/licenses/by-nc/4.0/.

\section{ORCID iDs}

Minhazul Abedin http://orcid.org/0000-0002-4783-3750

Farah Naz Rahman http://orcid.org/0000-0002-2265-2742

Shagoofa Rakhshanda http://orcid.org/0000-0001-9172-7042

Lauren M Fletcher http://orcid.org/0000-0002-6026-5823

Saidur Mashreky http://orcid.org/0000-0001-7892-798X

\section{REFERENCES}

1 WHO. WHO coronavirus (COVID-19) dashboard. Available: https:// covid19.who.int/ [Accessed 6 May 2021].

2 Abrams EM, Szefler SJ. COVID-19 and the impact of social determinants of health. Lancet Respir Med 2020;8:659-61.

3 WHO. Social determinants of health.. Available: https://www.who.int/ health-topics/social-determinants-of-health\#tab=tab_1 [Accessed 6 May 2021].

4 Marmot M, Allen JJ. Social determinants of health equity. Am J Public Health 2014:104 Suppl 4:S517-9.

5 The Health Foundation. Preventable deaths and deprivation. Available: https://www.health.org.uk/news-and-comment/chartsand-infographics/preventable-death-and-deprivation [Accessed 14 Apr 2021].

6 WHO. Part two. The urgent need for action, 2015. WHO. Available: http://www.who.int/chp/chronic_disease_report/part2_ch2/en/ [Accessed 15 Apr 2021].

7 Deaths involving COVID-19 by local area and socioeconomic deprivation. Office for national statistics. Available: https://www.ons. gov.uk/peoplepopulationandcommunity/birthsdeathsandmarriages/ deaths/bulletins/deathsinvolvingcovid19bylocalareasanddeprivation/ deathsoccurringbetween 1 marchand17april [Accessed 25 Apr 2021].

8 Adhikari S, Pantaleo NP, Feldman JM, et al. Assessment of community-level disparities in coronavirus disease 2019 (COVID-19) infections and deaths in large US metropolitan areas. JAMA Netw Open 2020;3:e2016938.

9 Lower income people, new immigrants at higher COVID-19 risk in Toronto, data suggests. CBC news. Available: https://www.cbc.ca/ news/canada/toronto/low-income-immigrants-covid-19-infection-1. 5566384 [Accessed 25 Apr 2021].

10 Millett GA, Jones AT, Benkeser D, et al. Assessing differential impacts of COVID-19 on black communities. Ann Epidemiol 2020;47:37-44.

11 Baqui P, Bica I, Marra V, et al. Ethnic and regional variations in hospital mortality from COVID-19 in Brazil: a cross-sectional observational study. Lancet Glob Heal 2020;8:e1018-26.

12 Martins-Filho PR, Araújo BCL, Sposato KB, et al. Racial disparities in COVID-19-related deaths in Brazil: black lives matter? J Epidemio 2021;31:239-40.

13 Wong DWS, Li Y. Spreading of COVID-19: density matters. PLoS One 2020;15:e0242398.

14 Coronavirus: in dense Bangladesh, social distancing a tough task. Coronavirus pandemic news. Al Jazeera. Available: https://www. aljazeera.com/news/2020/3/20/coronavirus-in-dense-bangladeshsocial-distancing-a-tough-task [Accessed 25 Apr 2021].

15 For India's slum dwellers, social distancing will be impossible during Covid-19 lockdown. CNN. Available: https://edition.cnn.com/2020/ 03/30/india/india-coronavirus-social-distancing-intl-hnk/index.html [Accessed 25 Apr 2021]

16 Kawachi I, Berkman LF. Social Capital, Social Cohesion, and Health. In: Social epidemiology. Oxford University Press, 2015: 290-319.

17 Ahmed SAKS, Ajisola M, Azeem K, et al. Impact of the societal response to COVID-19 on access to healthcare for non-COVID-19 health issues in slum communities of Bangladesh, Kenya, Nigeria and Pakistan: results of pre-COVID and COVID-19 lockdown stakeholder engagements. BMJ Glob Health 2020;5:e003042.

18 Shadmi E, Chen Y, Dourado I, et al. Health equity and COVID-19: global perspectives. Int J Equity Health 2020;19:104.

19 Oliver A, Mossialos E. Equity of access to health care: outlining the foundations for action. $J$ Epidemiol Community Health 2004;58:655-8.

20 Bhatt J, Bathija P. Ensuring access to quality health care in vulnerable communities. Acad Med 2018;93:1271-5.

21 Access to health services. Healthy people 2020. Available: https:// www.healthypeople.gov/2020/topics-objectives/topic/socialdeterminants-health/interventions-resources/access-to-health [Accessed 15 Apr 2021].

22 Health disparities by race and ethnicity. Center for American progress. Available: https://www.americanprogress.org/issues/ race/reports/2020/05/07/484742/health-disparities-race-ethnicity/ [Accessed 23 Apr 2021].

23 Buchmueller TC, Levinson ZM, Levy HG, et al. Effect of the affordable care act on racial and ethnic disparities in health insurance coverage. Am J Public Health 2016;106:1416-21.

24 In U.S., 14\% with likely COVID-19 to avoid care due to cost. Available: https://news.gallup.com/poll/309224/avoid-care-likelycovid-due-cost.aspx [Accessed 23 Apr 2021].

25 Transmission of SARS-CoV-2: implications for infection prevention precautions. Available: https://www.who.int/news-room/ commentaries/detail/transmission-of-sars-cov-2-implications-forinfection-prevention-precautions [Accessed 23 Apr 2021].

$26 \mathrm{Sohn} \mathrm{H}$. Racial and ethnic disparities in health insurance coverage: dynamics of gaining and losing coverage over the life-course. Popul Res Policy Rev 2017;36:181-201.

27 COVID-19 racial disparities in testing, infection, hospitalization, and death: analysis of epic patient data. KFF. Available: https://www.kff. org/coronavirus-covid-19/issue-brief/covid-19-racial-disparitiestesting-infection-hospitalization-death-analysis-epic-patient-data/ [Accessed 25 Apr 2021].

28 Risk for COVID-19 infection, hospitalization, and death by race/ ethnicity. CDC. Available: https://www.cdc.gov/coronavirus/2019 ncov/covid-data/investigations-discovery/hospitalization-death-byrace-ethnicity.html [Accessed $25 \mathrm{Apr}$ 2021].

29 Shahbazi F, Khazaei S. Socio-Economic inequality in global incidence and mortality rates from coronavirus disease 2019: an ecological study. New Microbes New Infect 2020;38:100762.

30 Seligman B, Ferranna M, Bloom DE. Social determinants of mortality from COVID-19: a simulation study using NHANES. PLoS Med 2021;18:e1003490.

31 Karmakar M, Lantz PM, Tipirneni R. Association of social and demographic factors with COVID-19 incidence and death rates in the US. JAMA Netw Open 2021:4:e2036462.

32 Baumer T, Phillips E, Dhadda A, et al. Epidemiology of the first wave of COVID-19 ICU admissions in South Wales-The interplay between ethnicity and deprivation. Front Med 2020;7:569714.

33 Indseth T, Grøsland M, Arnesen T, et al. COVID-19 among immigrants in Norway, notified infections, related hospitalizations and associated mortality: a register-based study. Scand J Public Health 2021;49:48-56

34 Germain S, Yong A. COVID-19 highlighting inequalities in access to healthcare in England: a case study of ethnic minority and migrant women. Fem Leg Stud 2020;28:301-10.

35 Szczepura A. Access to health care for ethnic minority populations. Postgrad Med J 2005;81:141-7.

36 Paremoer L, Nandi S, Serag H, et al. Covid-19 pandemic and the social determinants of health. BMJ 2021;372:n129.

37 Page MJ, McKenzie JE, Bossuyt PM, et al. The PRISMA 2020 statement: an updated guideline for reporting systematic reviews. BMJ 2021;372:n71

38 Social determinants of health. Healthy people 2020. Available: https://www.healthypeople.gov/2020/topics-objectives/topic/socialdeterminants-of-health [Accessed 15 Apr 2021]

39 PROGRESS-Plus. Cochrane equity. Available: https://methods. cochrane.org/equity/projects/evidence-equity/progress-plus [Accessed 9 May 2021]

40 Ovid database guide. Available: https://ospguides ovid .com/ OSPguides/medline.htm [Accessed 15 Apr 2021].

41 Ottawa Hospital Research Institute. The Newcastle-Ottawa scale (NOS) for assessing the quality of nonrandomised studies in meta-analyses. Available: http://www.ohri.ca/programs/clinical epidemiology/oxford.asp [Accessed 3 Apr 2021]

42 Campbell M, McKenzie JE, Sowden A, et al. Synthesis without metaanalysis (SWiM) in systematic reviews: reporting guideline. BMJ 2020;368:16890. 